\title{
Drill-Locate-Drill Algorithm for Diagnostic Reasoning in Psychiatry
}

\author{
Irosh Fernando and Frans A. Henskens
}

\begin{abstract}
This paper introduces a top-down algorithm for diagnosing psychiatric illnesses. It is based on the conceptualisation of diagnostic categories, diagnosis, and symptoms as a hierarchical model. The algorithm assumes that there exist a few close-ended clinical questions that can be used during clinical interview to rule in and rule out diagnostic categories, diagnoses and their symptoms. Compared to a more exhaustive bottom-up and recursive algorithm, which the authors have previously introduced, this algorithm has the advantage of being easy to implement requiring a less extensive knowledgebase. It is expected the algorithm will be used as a useful screening tool that increases the detection of psychiatric disorders, which are common but unfortunately currently under-diagnosed.
\end{abstract}

Index Terms-Diagnosis, diagnostic algorithm, psychiatry, screening tool.

\section{INTRODUCTION}

Psychiatric disorders have been recognised as some of the most disabling illnesses causing a significant level of burden on patients and their families [1]. Unfortunately, psychiatric disorders are often unrecognized [2]. Particularly, according to the World Health Organization (WHO), in those who have a psychiatric disorder less than half are identified by doctors [3]. Compared to general medical disorders, diagnosing psychiatric disorders can be challenging due to highly subjective and ambiguous nature of their symptoms and the lack of objective diagnostic tests.

Diagnostic decision support tools can be very useful in psychiatry as they can potentially save clinical time and resources, and also improve the reliability of the diagnostic reasoning process.

Even though some of the well-known computer assisted diagnostic tools such as Composite International Diagnostic Interview (CIDI) are available in psychiatry [4], they can be viewed as a set of standard checklists of symptoms. Expert diagnostic reasoning is a dynamic process, which is directed by the relevance and the importance of the information required, and an experienced clinician can change the direction of the interview process in order to seek the most relevant and important clinical information during the limited available clinical time. The standard symptom checklist represents a static approach, which can be time consuming and lacks customisation in accordance with the relevance and importance of the information required.

This paper briefly discusses some of the previous related

Manuscript received September 7, 2013; revised October 25, 2013.

The authors are with the School of Electrical Engineering \& Computer Science, University of Newcastle, NSW 2308, Australia (phone: +61 423 281 664; e-mail: irosh.fernando@uon.edu.au, frans.henskens@newcastle.edu.au). approaches, and introduces a top-down algorithm, which is currently being developed as a solution.

\section{RELATED APPROACHES}

Whilst medical expert systems strive to mimic the dynamic reasoning process, developing practical and useful medical expert systems can be very challenging. Even though the realisation of medical expert systems was one of the earliest goals of the AI community, some of the largest projects undertaken, for example, INTERNIST-I and CADUCEUS, have been unsuccessful [5].

As the authors previously described, possible reasons for such failures include:

1) Taking too generalised an approach / lack of customisation according to the subdomain of expertise;

2) Difficulty in developing conceptual and formal models that capture the complexity and depth of the clinical reasoning process [6]; and

3) Not paying adequate attention to socio-organisational aspects of the expert system being developed [7].

As a remedy the authors introduced a software process model for expert system development known as the Collaborative and Layered Approach (CLAP), which was followed in developing the approach proposed in this paper [8].

The authors previously introduced a general approach to medical diagnostic reasoning which is bottom-up and recursive in nature [9]. This general approach was also adopted for diagnostic reasoning in psychiatry [10]. However, the alternative approach proposed in this paper has the advantage of being tailor-made to the clinical nature of psychiatry, and is expected to be more efficient and easy to implement because it is highly structured and top-down.

\section{CONCEPTUAL Model}

All psychiatric disorders have been neatly classified into a small number of diagnostic categories [11]. In clinical interviews it is often possible to rule in and rule out the possibility of someone having a psychiatric disorder in one, or more than one, category. In a similar manner, whilst each diagnostic category has a relatively small number of disorders, it is possible to use a small number of key questions to clarify whether it is likely that someone had/has a particular psychiatric disorder of a given category. If it is found to be likely that someone has a particular psychiatric disorder or that someone had a psychiatric disorder in the past, then specific questions can be asked to elicit the expected symptoms of the likely psychiatric disorder. This top-down interview process is described in Fig. 1. 


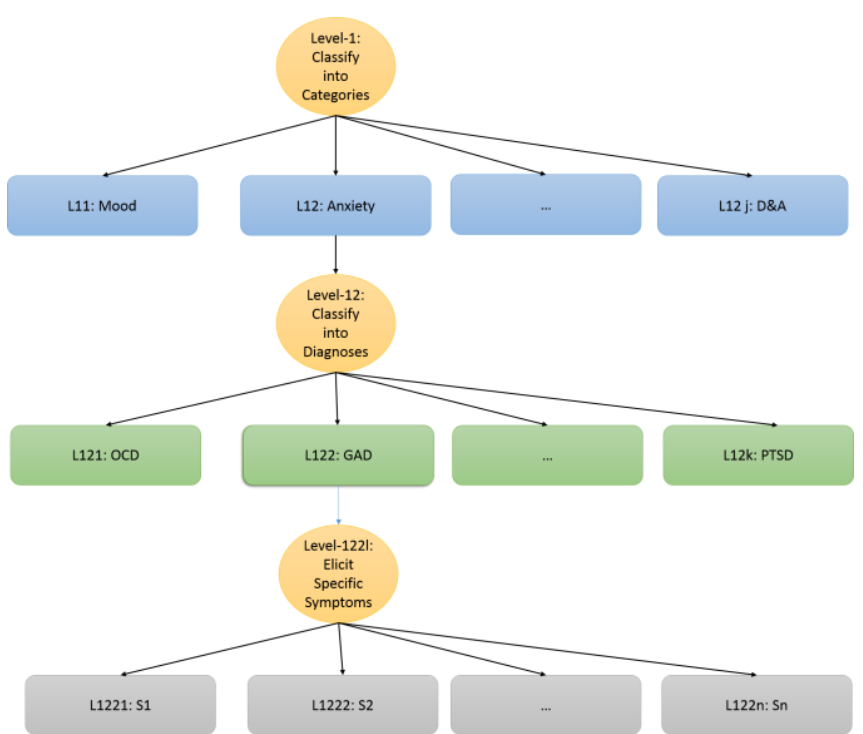

Fig. 1. Top-down diagnostic interview process (only the pathway towards Generalised Anxiety Disorder (GAD) is shown).

\section{FORMAL MODEL}

In formalising the conceptual model described above, let $C=\left\{c_{1}, c_{2}, \ldots\right\}$ be the set of all diagnostic categories; $c_{i}=$ $\left\{d_{i 1}, d_{i 2}, \ldots\right\}$ be the set of all the diagnoses in theith category; and $d_{i j}=\left\{s_{i j 1}, s_{i j 2}, \ldots\right\}$ be the set of all the symptoms related to $j$ th diagnosis in the $i$ th category.

Corresponding to $c_{i}$ there exists a set of clinical questions $Q\left(c_{i}\right)=\left\{q_{c_{i} 1}, q_{c_{1} 2}, \ldots\right\}$ that can be used during a clinical interview to ascertain $c_{i}$. In a similar way, corresponding to $d_{i j}$ there exists a set of clinical questions $Q\left(d_{i j}\right)=\left\{q_{d_{i j} 1}, q_{d_{i j} 2}, \ldots\right\}$ that can be used during a clinical interview to ascertain $d_{i j}$; corresponding to $s_{i j k}$, which is the $k$ th symptom of $j$ th diagnosis in $i$ th category, there exists a set of clinical questions $Q\left(s_{i j k}\right)=\left\{q_{s_{i j k} 1}, q_{s_{i j k} 2}, \ldots\right\}$ that can be used during a clinical interview to ascertain $s_{i j k}$.

Let $R(q) \in\{$ True, False $\}$, be the set of possible responses to question $q$ where $q \in\left\{Q\left(c_{i}\right) \cup Q\left(d_{i j}\right) \cup Q\left(s_{d_{i} k}\right)\right\}$.

Also, let $L\left(c_{i}\right), L\left(d_{i j}\right), L\left(s_{i j k}\right) \in\{$ True, False $\}$ be the set of possibilities of a patient meeting the criteria for $c_{i}, d_{i j}$ and $s_{i j k}$ respectively.

Then diagnostic rules are defined as:

$$
\begin{gathered}
E\left(c_{i}\right) \Rightarrow L\left(c_{i}\right), \\
E\left(d_{i j}\right) \Rightarrow L\left(d_{i j}\right), \text { and } \\
E\left(S_{i j k}\right) \Rightarrow L\left(s_{i j k}\right)
\end{gathered}
$$

where $E\left(c_{i}\right), E\left(d_{i j}\right)$ and $E\left(S_{i j k}\right)$ are logical expressions composed of different $R(q)$ using the standard logical operators.

The following example illustrates the above-described formal model:

$$
\begin{gathered}
c_{1}=\text { Mood Disorders } \\
d_{11}=\text { Major Depressive Disorder }
\end{gathered}
$$

$$
\begin{aligned}
& s_{111}=\text { Depressed Mood } \\
& s_{112}=\text { Loss of interest }
\end{aligned}
$$$$
s_{113}=\text { Loss of appetite or loss of weight }
$$$$
s_{114}=\text { Sleep difficulties }
$$

$s_{115}=$ Feeling worthless or excessive guilt

$q_{s_{111} 1}=$ Haveyoubeenexperiencingdepressedmood $?$

$$
\begin{gathered}
q_{s_{112} 1}=\text { Haveyoulostinterest } ? \\
q_{s_{113} 1}=\text { Haveyoulostappetiteorweight? }
\end{gathered}
$$

$q_{s_{114} 1}=$ Haveyoubeenexperiencingsleepdifficulties $?$

$q_{s_{115} 1}$

= Haveyoubeenfeelingworthlessorexcessiveguilt?

$$
\begin{aligned}
& E\left(d_{11}\right)=R\left(q_{s_{111} 1}\right) \wedge R\left(q_{s_{112} 1}\right) \\
& \wedge\left(R\left(q_{s_{113} 1}\right) \vee R\left(q_{s_{114} 1}\right) \vee R\left(q_{s_{115} 1}\right)\right) \\
& E\left(d_{11}\right) \Longrightarrow L\left(d_{11}\right)
\end{aligned}
$$

\section{ALGORITHM}

As described in the conceptual model and the formal model, the aim of the algorithm is to narrow down the search space at each step in the following order:

1) Locate the likely category/ categories of diagnoses;

2) Locate the likely diagnosis or diagnoses in a selected category; and

3) Check if the symptoms of a selected diagnosis are present in the patient.

The term 'drill-locate-drill' was used to describe this algorithm since the first step involves 'drilling' one level down to diagnostic categories, and then locating the most likely category of diagnoses. Once a likely diagnostic category is located, the next step involves 'drilling' one level of diagnoses further down, and then locating the most likely diagnosis or diagnoses. The final step involves 'drilling' one more level down to symptoms of diagnoses, and then locating the symptoms that are present in the patient. The process of 'locating' a likely category or diagnosis involves invoking a function, which returns the Boolean value 'true' if the related category/diagnoses is likely, and 'false' otherwise.

Fig. 2 describes the algorithm. The functions $\operatorname{checkCategory}(i)$, checkDiagnosis $(i, j)$, and elicitSymptomsOfDiagnosis $(i, j, k)$ elicit responses to questions related to the $i$ th diagnostic category, $j$ th diagnosis, and $k$ th symptom respectively, and return a Boolean value after evaluating the respective logical expressions. In a similar manner, the function evaluateDiagnosticCriteria( $i, j$, Symptoms []) returns a Boolean value after evaluating the respective logical expressions related to ascertaining the $j$ th diagnosis in the $i$ th diagnostic category. In doing so, it uses the responses to the 
relevant questions that are stored in the array Symptoms[].

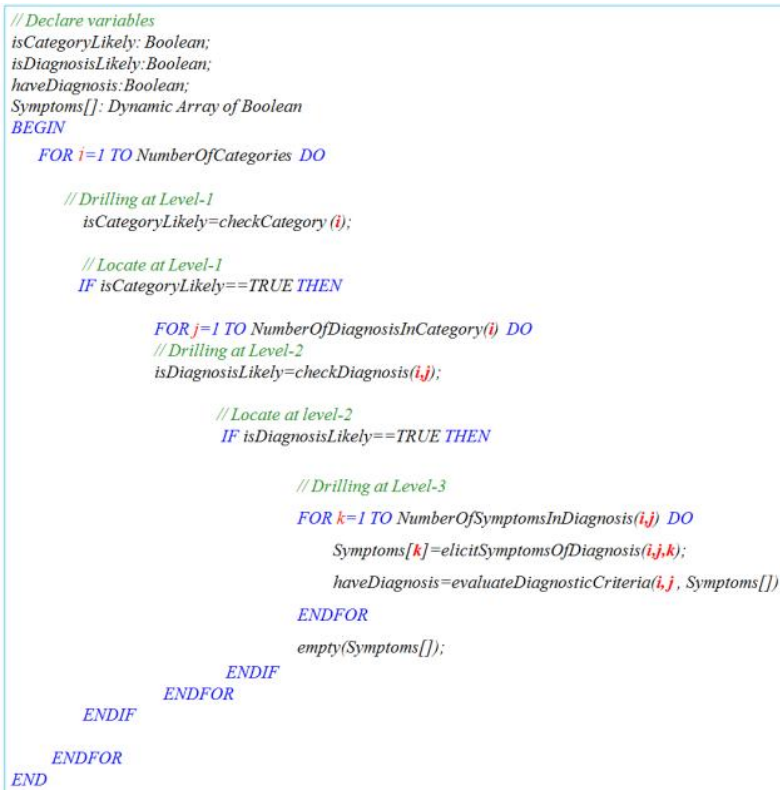

\section{IMPLEMENTATION}

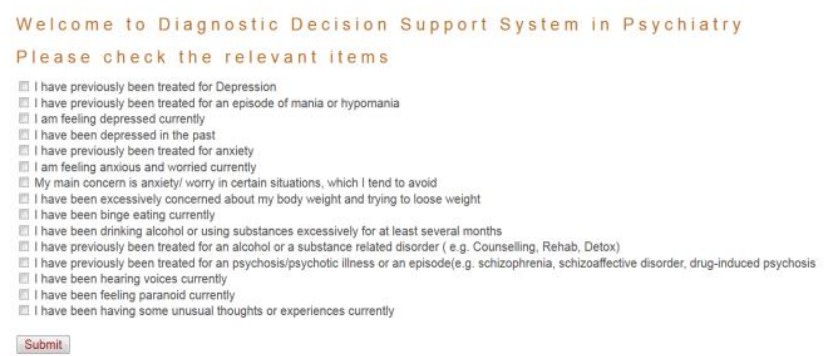

Fig. 3. User interface for locating diagnostic categories.

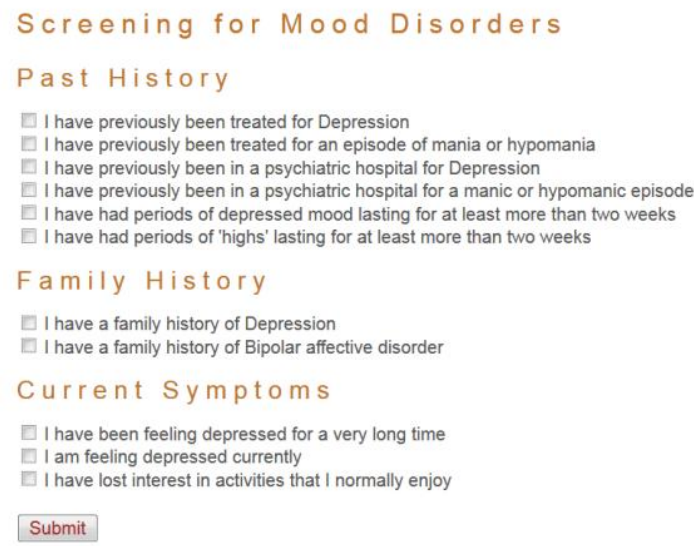

Fig. 4. User interface for locating diagnosis, depression.

This algorithm is currently being implemented using an evolutionary prototyping approach according to the CLAP software process model [8], particularly with the view of identifying the screening questions that can be used for reliably locating each category, and diagnoses within each category. It has been implemented as a web-based system using Java web technology [12], GlassFish server [13] and MySQL server [14].

The diagnostic categories included in the initial prototype included: mood disorders; anxiety disorders; eating disorders; psychotic disorders; and drug and alcohol related disorders.

Implementation of the user interface for 'drilling' at level 1 (i.e. diagnostic categories) with the related key questions are shown in Fig. 3.

As an example, suppose the category mood disorders was located, then the user interface for 'drilling ' down to level 2 (i.e. diagnoses) is shown in Fig. 4.

Similarly, next suppose the diagnosis depression was located, then the user interface for 'drilling' down to level 3 (i.e. symptoms) is shown in Fig. 5.

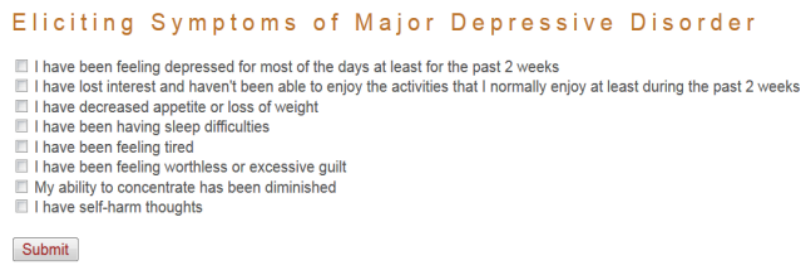

Fig. 5. User interface for eliciting symptoms of depression.

\section{DISCUSSION}

As stated under the formal model, the algorithm described in this paper relies on two key assumptions, and it is possible that there can be atypical clinical situations in which these assumptions are violated. Also, one of the main limitations of a top-down approach is the defined scope of the search, and it is possible that there can be clinical situations that may fall outside of this scope (i.e. medical disorders) and therefore can be missed.

The advantage of the algorithm is its simplicity, which is achieved at the cost of this risk. Therefore, the drill-locate-drill algorithm and the accompanying tool is intended only as a decision support tool for assisting clinicians, and is not intended as a substitute for the clinical reasoning skills of clinicians. By way of comparison, the authors' previously described algorithm[10]is more comprehensive but also more complex and difficult to implement because of the larger knowledgebase it requires.

Particularly in psychiatry, case formulation (i.e. the narrative account describing why a particular patient became unwell at the time the illness occurred) is often more clinically important than the diagnosis, and can be a more difficult problem to solve. Use of the drill-locate-drill algorithm and tool presented in this paper can support clinicians to apply their limited clinical time solving more complex problems, such as case formulation, by automating and saving the time it requires for diagnostic reasoning.

Selection of screening questions that have the highest diagnostic sensitivity, and phrasing them in such a way that patients can easily understand them, can be challenging and often requires a 'trial and error' approach. Therefore, ongoing system development and refinement necessarily requires an evolutionary prototyping approach.

\section{CONCLUSION}

This paper introduced a top-down algorithmtermed 
drill-locate-drill for diagnosing common psychiatric disorders. The algorithm is currently being implemented and evaluated using an evolutionary prototyping approach. The algorithm has the advantage of being easy to implement, and efficiently narrows down the diagnostic search space. However, because of the top-down nature of the algorithm, it is possible for it to miss some of the atypical presentations of psychiatric disorders. It should therefore not be considered as a substitute for the reasoning skills of an experienced clinician.

An implementation of this algorithm can be used as a screening tool for enhancing the detection of psychiatric disorders, and also for managing psychiatric referrals and triaging them. Such a tool that implements the algorithm has the potential advantage of improving the reliability of psychiatric diagnoses using standard diagnostic criteria such as DSM-V[11], and saving precious clinical time and resources by automating one aspect of clinical reasoning. The authors expect to further improve and enhance the algorithm and its implementation using feedback from the evolutionary prototyping approach.

\section{REFERENCES}

[1] W. W. Eaton, S. S. Martins, G. Nestadt, O. J. Bienvenu, D. Clarke, and P. Alexandre, "The burden of mental disorders," Epidemiol Review, vol. 30, pp. 1-14, 2008.

[2] S. Kunen, R. Niederhauser, P. O. Smith, J. A. Morris, and B. D. Marx, "Race disparities in psychiatric rates in emergency departments," Journal of Consulting and Clinical Psychology, vol. 73, no. 1, pp. 116-126, 2005.

[3] World Health Organisation. (2013). Gender and women's mental health: gender disparities and mental health: The facts. [Online] Available:

http://www.who.int/mental_health/prevention/genderwomen/en/

[4] L. N. Robins et al., "The composite international diagnostic interview: An epidemiologic instrument suitable for use in conjunction with different diagnostic systems and in different cultures," Arch Gen Psychiatry, vol. 45, pp. 1069-77, Dec 1988.

[5] D. A. Wolfram, "An appraisal of INTERNIST-I," Artificial Intelligence in Medicine, vol. 7, pp. 93-116, 1995.

[6] I. Fernando, F. A. Henskens, and M. Cohen, "A domain specific conceptual model for a medical expert system in psychiatry, and a development framework," presented at the IADIS e-Health International Conference, Rome, Italy, 2011.

[7] R. L. Wears and M. Berg, "Computer technology and clinical work still waiting for godot," Journal of the American Mecical Association, vol. 293, no. 10, pp. 1261-1263, 2005

[8] I. Fernando, F. Henskens, and M. Cohen, "A Collaborative and Layered Approach (CLAP) for medical expert system development: a software process model," in Proc. IEEE/ACIS 11th International Conference on Computer and Information Science (ICIS12), 2012, pp. 497-502.

[9] I. Fernando, F. Henskens, and M. Cohen, "An approximate reasoning model for medical diagnosis," in Software Engineering, Artificial
Intelligence, Networking and Parallel/Distributed Computing, vol. 492, R. Lee, Ed. Springer International Publishing, 2013, pp. 11-24.

[10] I. Fernando, M. Cohen, and F. Henskens, "A systematic approach to clinical reasoning in psychiatry," Australasian Psychiatry, vol. 21, pp. 224-230, 2013.

[11] American Psychiatric Association, Diagnostic and Statistical Manual of Mental Disorders: DSM-5, American Psychiatric Publishing Incorporated, 2013.

[12] D. Nourie. (2006). Java technologies for web applications. [Online]. Available:

http://www.oracle.com/technetwork/articles/javase/webapps-1-13879 4.html

[13] Oracle Corporation. (2013). Glassfish - World's first Java EE 7 application server. [Online]. Available: https://glassfish.java.net

[14] Oracle, MySQL Reference Manual, 5.5 ed. Oracle, 2012.

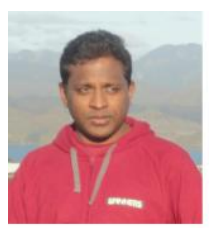

Irosh Fernando was born in Negombo, Sri-Lanka. He studied medicine at the University of Colombo, gaining his MBBS in 2000, and then MD (Psychiatry) in 2006. He also completed the British Computer Society Examination in 1998, and graduated with MPhil in computer science from the Open University of Colombo in 2008.

Whilst working as a medical practitioner since 2001, and he also worked as a visiting lecturer in computer science for IDM Negombo Colombo Campuses during 1996 to 2004. He is currently a staff specialist psychiatrist at Calvary Mater Hospital, Hunter New England Area Health Service, NSW, Australia, and also a Ph.D. candidate in Computer Science at the School of Electrical Engineering and Computer Science at the University of Newcastle, Australia.

Dr Fernando is a fellow of the Royal Australian and New Zealand College of Psychatrists (RANZCP), and a member of the Institute of Electrical and Electronic Engineers (IEEE); the Australian Computer Society (ACS); the British Computer Society (BCS); and the American Mathematical Society (AMS).

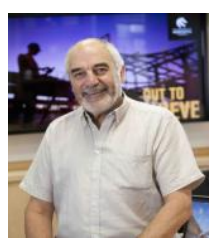

Frans A. Henskens was born in Newcastle, Australia in 1951. He studied mathematics and education, graduating with BMath in 1975 and DipEd in 1976. He later studied computer science, graduating DipCompSc in 1986 and a Ph.D. in 1992, all these studies at the University of Newcastle, Australia.

He has worked the University of Newcastle, Sydney University, and Universitaat Ulm, and is now an associate professor, deputy head of School of Electrical Engineering and Computer Science, and assistant dean (IT) of the Faculty of Engineering \& Built Environment at the University of Newcastle, Australia. He has over 130 peer reviewed publications, and has attracted almost $\$ 7$ million of research funding. His research interests include distributed computing, computer systems, and software engineering. His current research concentrates on improving the way we use the Internet, support for professional decision making, and the role of computer support in medical areas such as health behaviour, mental health, cancer, and sleep disorders.

Prof. Henskens received a Fellowship under the Baden-Württemberg Visiting Scholars Program in Ulm, Germany, 2002; the Vice Chancellor's Award for Teaching Excellence in 2007; and the Australian College of Educators NSW Quality Teaching Award in 2007. 